\title{
How complex are the contracts offered by health plans?
}

\author{
Pau Olivella • Marcos Vera-Hernandez
}

Received: 10 October 2008 / Accepted: 7 April 2010 / Published online: 4 May 2010

(C) The Author(s) 2010. This article is published with open access at Springerlink.com

\begin{abstract}
When health plans compete under adverse selection, the competitive equilibrium set of contracts is unique. However, the allocation of these contracts among health plans is undetermined. We show that three health plans suffice to sustain an equilibrium where each health plan offers a single contract and attracts a single type of agent (full specialization). We also show that this equilibrium can be ruled out by introducing any horizontal differentiation, and that if in equilibrium each health plan attracts all types of agents, at least one of the health plans must do so through a menu of contracts.
\end{abstract}

Keywords Health plans competition · Adverse selection · Differentiation

JEL Classification $\quad$ D82 $\cdot \mathrm{I} 11 \cdot \mathrm{L} 13$

\section{Introduction}

Health plans are a popular way to deliver health care. Health plans receive a fee per affiliate in exchange for providing him/her directly with the required health care. They are very important in the US as well as in some European countries. The main purpose of our investigation is to explain why in reality it is often the case that health plans

\footnotetext{
P. Olivella $(\varangle)$

Departament d'Economia i d'Historia Economica and MOVE, Universitat Autònoma de Barcelona, 08193 Bellaterra, Spain

e-mail: pau.olivella@uab.es

M. Vera-Hernandez

Department of Economics, University College London, Gower Street,

London WC1E 6BT, UK

e-mail: uctpamv@ucl.ac.uk
} 
offer menus of contracts, despite the fact that this is not a necessary implication of the usual model of competitive screening.

Indeed, since Rothschild and Stiglitz (1976) seminal paper it is well understood that one should observe two contracts in equilibrium - one intended for the low risks and the other for the high risks. However, this does not mean that each health plan offer both contracts, as in a menu. ${ }^{1}$ While menus are a possibility, a situation where some health plans only offer the contract intended for the high risk individuals and the rest only offer the contract intended for the low risk individuals also constitutes an equilibrium (which we refer to as "full specialization"). It is important to highlight that this equilibrium exists even if one allows health plans to offer menus of contracts. In other words, it turns out that the same equilibrium set of available contracts emerges independently of whether health plans are allowed to offer menus of contracts or just single contracts. ${ }^{2}$ In this sense, the complexity of the offers by each plan is indeterminate. Our main contribution is to prove that this conclusion doe not extend to any horizontal differentiation. ${ }^{3}$ We are in fact able go beyond this result. We can prove that it cannot be an equilibrium that health plans attract all types of agents with a single contract each, in other words, at least one health plan must offer a menu of contracts in equilibrium. This may explain why in reality it is often the case that health plans offer menus of contracts. For instance, in Washington, DC (zip code 20001) "Bravo Classic" offers more comprehensive drug coverage than "Bravo Gold" but "Bravo Gold" offers more comprehensive dental coverage. ${ }^{4}$

In order to fully grasp the importance of our result, we need to address the difference between contract complexity within and across risk classes. A risk class is the set of individuals (usually defined by the value of their demographics such as age and gender) that can choose among a set of contracts. In many contexts, "open enrolment rules" apply, meaning that the risk class is the entire population. In other contexts,

\footnotetext{
${ }^{1}$ Even if each and all health plans offer a menu of contracts, it is not necessarily true that all health plans obtain the same share of both types. Any other distribution of types is also an equilibrium. For instance, a health plan offering the equilibrium menu may monopolize all low risk individuals and attract a single high risk individual while another health plan obtains the rest of high risks; and so on.

2 However, this matters for the existence of equilibrium. When firms are allowed to offer menus of contracts, the parameter set for which an equilibrium exist is smaller than if firms can only offer single contracts. However, existence is addressed only in passing in the present work.

3 As in Ellis (1998), the model does not preclude interpreting distance as a consumer's disutility from seeking a certain style of health care.

${ }^{4}$ Other examples of menus include copayments at the point of consumption, which we assume away in our analysis. However, these copayments could be thought as a decrease in the quality of the service. In any case, they are quite small when compared to the total cost of care. For instance, in San Francisco (zip code 94102), "SecureHorizons MedicareDirect" offers "Plan 5" and "Rx Plan 56". The former has lower copayments but the latter has a wider coverage of prescription drugs. The same can be said about "Essential" and "Plan 1" both offered by "AARP MedicareComplete" in San Diego (zip code 92101). The insurer "Humana" offers both "Choice PPO" and "Gold Plus HMO" in Boston (zip code 60601). "Choice PPO" has higher copayments than "Gold Plus HMO" but allows to choose providers outside their network. Its coverage of dental services is more comprehensive than the dental coverage offered by "Gold Plus HMO" but it does not cover vision services unlike "Gold Plus HMO". "Gold Plus HMO" has a wider coverage of prescription drugs. See http://www.medicare.gov/MPPF/Include/DataSection/ Questions/GeneralQuestions.asp. Consistently with our analysis, in any of these examples, insures do not need to pay any premium in addition to their normal Medicare Part B premium.
} 
risk classes are more narrowly defined because insurance companies can legally offer different contracts to individuals with different value of their demographics. It is important to understand that the results of our model hold within a risk class, no matter how narrowly or widely defined it is. This means that we are addressing the question of multiplicity of contracts in the menu offered to each class. It is in this sense that we use the word "complexity" in the title of our paper. This is different from multiplicity of contracts across risk classes, usually known as "risk classification" or "categorization" (Crocker and Snow 2000), under which insurance companies offer different contracts to individuals with different demographics but the same contract to everyone with the same demographics.

Our model is addressed to deal with situations where the third party payer pays the capitation rate in full (or most of it), so that health plans cannot use the part of the capitation rate charged to the individual (premium) as a tool to screen individuals. This includes Medicare and Medicaid in the US, the provision of health care of those under 18 years of age in The Netherlands, the health insurance system in Colombia after the 1993 reform, and the system providing health care to civil servants in Spain. ${ }^{5}$

Our results complement what we have learned from the literature dealing with markets where both adverse selection and horizontal differentiation are present (Jack 2006; Olivella and Vera-Hernández 2007; Biglaiser and Ma 2003; Villas-Boas and Schmidt-Mohr 1999). This literature has restricted attention to a very particular form of equilibria: the symmetric separating equilibrium in which the same menu of contracts is used by all health plans to separate risk types. ${ }^{6}$ This strategy for finding an equilibrium, which indeed simplifies the analysis, leaves us with the question of whether other equilibria may exist. Our work provides a partial answer to this strategy, as it rules out two alternative equilibrium configurations.

The model of horizontal differentiation mostly used in the aforementioned literature is one where only two health plans exist in the market (one at each extreme of the Hotelling line). This raises the question of whether the elimination of the fully specialized equilibrium is just a result of limiting (to 2) the number of health plans in the market, rather than the effect of horizontal differentiation. Indeed, when the number of health plans coincides with the number of types, full specialization is not an equilibrium even in the absence of horizontal differentiation. ${ }^{7}$ An important contribution of our analysis is to show that three health plans are enough to sustain the equilibrium with full specialization in the model without differentiation. Therefore, in order to elicit the effect of horizontal differentiation alone, we use an extension of the Hotelling model to encompass more than two health plans and we show that full

\footnotetext{
5 Civil servants in Spain are allowed (paradoxically) to opt out of the compulsory national public health system and to choose among competing private health plans (Pellisé 1994). This system is referred to as MUFACE, and we return to it in a footnote in the last section.

6 Ellis and McGuire (2007), Frank et al. (2000), Ma (2004), and Olivella and Schroyen (2009), among others, also study the market for health plans with horizontal differentiation, but they assume symmetric information among the parties. They instead focus on an empirical measure of the distortions caused by cream skimming and dumping (risk selection). Incidentally, these authors also focus on the symmetric equilibrium.

7 In this respect, Villeneuve (2003) was the first to point out that, in order to extend the results in Rothschild and Stiglitz to a market with a fixed number of firms, one needs to have at least three firms.
} 
specialization is not an equilibrium in this model. We insist that this result holds for any positive transportation costs, however small. To extend the Hotelling model to more than two health plans, we use the spokes model first characterized by Chen and Riordan (2007). ${ }^{8}$

As mentioned above, our results complement the literature of competition among health plans. Olivella and Vera-Hernández (2007) characterize the symmetric separating equilibrium where each and all health plans offer the same menu of incentive compatible contracts. They show that, despite the introduction of differentiation among health plans, an important feature of the equilibrium in the homogenous product model is retained. Namely, the contract that is aimed at low risks contains a distortion. Those services that are relatively used more intensely by the high risks will be underprovided while those services that are used with the same intensity across all population will be overprovided. In contrast, the equilibrium under differentiation presents two novel features. First, cross subsidization may exist between the low and the high risks. In other words, health plans will obtain —in equilibrium - positive profits from each low risk and will suffer losses from each high risk. ${ }^{9}$ This is novel because in the unique separating set of equilibrium contracts under homogeneous product, each affiliate brings zero profits irrespective of his/her type. This also partly explains why it is possible to sustain an equilibrium with full specialization in the homogenous product scenario. More importantly, the competitive equilibrium may fail to be second best. Olivella and Vera-Hernández (2007) show that this will be the case precisely when the equilibrium presents cross subsidization. Jack (2006) analyzes the optimal adjustment of capitation rates in the presence of differentiation. He shows that the results derived by Glazer and McGuire (2000) in the homogenous product scenario are robust. Namely, if the capitation rate can be adjusted according to some observable (say age), then the capitation that an old individual brings should more than cover his/her expected expenses, while the opposite should hold true for the young. Biglaiser and Ma (2003) analyze the market for two services that can be either sold in a bundle or separately. Villas-Boas and Schmidt-Mohr (1999) focus on credit markets and study the case of local monopolies (less than full market coverage). Encinosa and Sappington (1997) also simultaneously consider adverse selection and a market imperfection, but the imperfection there is caused by the existence of fixed entry costs. Moreover, they directly assume that each plan offers a single contract. They conclude that a pooling equilibrium where low risks subsidize the high risks exists. In the same spirit, Newhouse (1996) argues that

\footnotetext{
8 An alternative, and quite standard, model with horizontal differentiation and more than two firms is Salop's circular city model (Salop 1979). We have opted for the spokes model because it is a simpler one to deal with asymmetric equilibria, which we have to confront when we address the issue of full specialization. Indeed, under full specialization some health plans offer the contract attracting low risks while the rest offer the contract attracting the high risks. Since in the Salop circle each firm is only directly competing with its two neighbors (one in each side), such asymmetries would lead us to a large number of cases. Instead, in the spokes model each firm is directly competing with the rest. Neither the spokes model nor the circular city model have ever been used to model a competitive screening model with differentiation. These models are generally used to study the effects of entry under symmetric information.

9 Profits are on average positive at each firm, but this is unsurprising given the introduction of market power.
} 
if introducing new contracts is not costless (commercialization costs) then a pooling equilibrium may exist. We assume away any such costs.

As for the issue of complexity per se, there are at least two strands of the literature that are somewhat related to our work. The first one argues that complexity itself could be a strategic variable. In this case, it is important to ascertain whether contract complexities are strategic complements or substitutes. This ties with the commercialization costs mentioned above. It is a challenge to empirically distinguish between strategic complementarities and the presence of unobserved heterogeneity in the size of these commercialization costs (Miravete 2009). The other strand addressing contract complexity asks whether this phenomenon could limit competition by raising switching costs (Frank and Lamiraud 2009). Notice however, that this last strand is concerned with the complexity of the full set of contracts outstanding in the market, not at each health plan.

The paper is organized as follows. In Sect. 2 we present the model and analyze the homogeneous product case as a benchmark. In Sect. 3 we analyze the game under product differentiation and show our main results. In Sect. 4 we discuss possible reasons why our results may not apply in some settings and conclude the paper. All proofs are in Appendix.

\section{The model}

We base our model on a particular case of the spokes model as formalized in Chen and Riordan (2007). ${ }^{10}$ This restricted version of the spokes model constitutes the simplest possible extension to the classic Hotelling model with extreme differentiation, which is the one that is mostly used in the literature. Our analysis considers $N$ health plans and two continua of agents (potential enrollees). Each health plan has one hospital. Each of the $N$ hospitals are located at the ends of each of the $N$ spokes $i=1,2, \ldots, N$ emanating from a common origin, as illustrated in Fig. 1. The spokes have length 1/2. We denote locations in each spoke with their distance to the extreme of the spoke. Hence health plans are located at point zero in each spoke. Health plan $i$ will be the health plan with a hospital located at the extreme of spoke $i$.

Agents are uniformly distributed on the spokes. Their total mass is one. Transportation costs are $t>0$ per unit of distance. Agents can be of two types, high risks or low risks, in the sense explained below. Each agent knows his type but this is unknown to the health plans. Plans do know that there is a proportion $\gamma$ (respectively, $1-\gamma$ ) of low (high) risks. A third party compensates each health plan at a rate $r>0$ per affiliated agent, regardless of the agent's type.

Health plans compete in the quality of the medical treatment offered. There are two illnesses, $\mathrm{M}$ and $\mathrm{N}$. The probability of suffering illness $\mathrm{M}$ is one. ${ }^{11}$ The probability of

\footnotetext{
$\overline{10}$ Namely, they allow some spokes to be empty while we assume that each an all spokes have a health plan.

11 This is just for simplicity. What is important is that all individuals face the same probability. Otherwise we would be facing a multidimensional screening competitive model, the solution of which for the perfectly competitive case is elusive. See for instance Villeneuve (2003).
} 


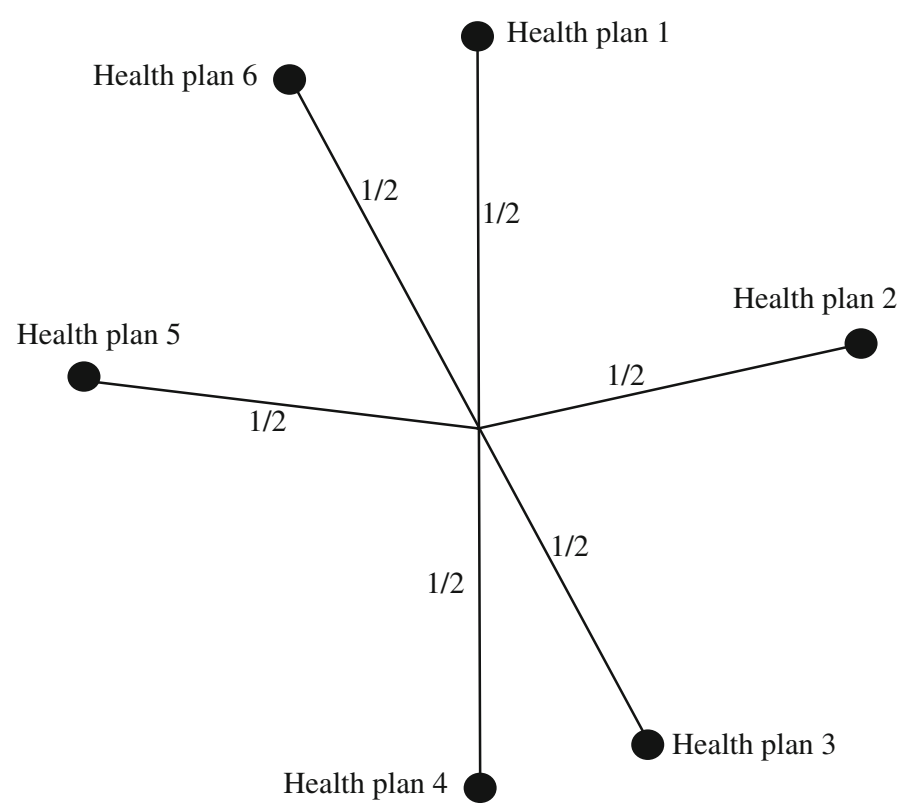

Fig. 1 The spokes model when $N=6$

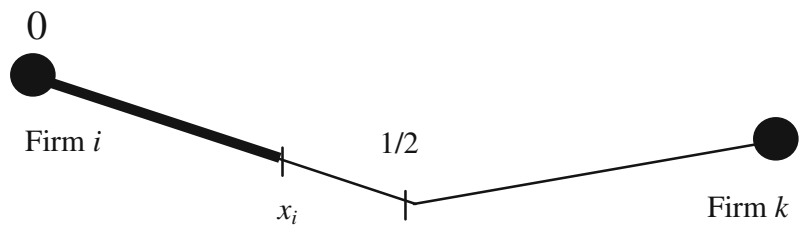

Fig. 2 Traveling distances for an individual who resides in spoke $i$ and whose alternative plan is $k$

suffering illness $\mathrm{N}$ is $p_{L}$ for a low risk agent, and $p_{H}$ for a high risk agent. We assume that $0<p_{L}<p_{H}<1$.

Irrespective of his type, each agent can enroll into exactly one of two pre-specified health plans: the one that is on his own spoke and some other plan that is ex-ante randomly and exogenously chosen. ${ }^{12}$ This is illustrated in Fig. 2, where the agent located at point $x_{i}$ in spoke $i$ in the network will be facing a choice between health plan $i$ and some other plan $k$ chosen by nature. His transportation costs are $t x_{i}$ if he enrolls health plan $i$ and $\left(\frac{1}{2}+\frac{1}{2}-x_{i}\right) t$ if he enrolls plan $k$. We refer to plan $k$ simply as this agent's "alternative plan". All health plans are equally likely to be selected as the alternative, so the probability of each is $1 /(N-1)$.

Letting $m_{i}$ and $n_{i}$ be the level of expenditures incurred by health plan $i$ in treating illness $\mathrm{M}$ and $\mathrm{N}$, respectively, the Von-Neumann-Morgenstern gross utility function

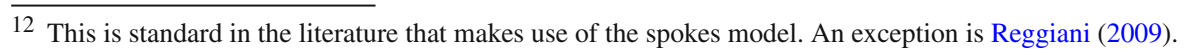


of an agent type $J$ affiliated to a health plan $i$ is

$$
U^{J}\left(m_{i}, n_{i}\right)=v\left(m_{i}\right)+p_{J} v\left(n_{i}\right) ; J=H, L, \text { and } i=1, \ldots, N .
$$

Expenditures $m_{i}$ and $n_{i}$ are only realized if agents are treated. These expenditures translate into treatment qualities, which are observable by agents. For simplicity, we have assumed that the function translating expenditures into qualities is the same for both treatments. All the arguments hereafter generalize to setting $v_{M}(z) \neq v_{N}(z)$ for some $z$. We assume that $v$ is twice-differentiable, increasing and concave.

\subsection{Health plans' contract choices}

We assume that both an agent's location and alternative health plan are non-contractible and hence the expenditure on medical treatment cannot depend on them (either because it is unobservable by the health plan, or because the law does not allow it). ${ }^{13}$ Therefore, health plan $i, i=1, \ldots, N$; chooses a menu of two contracts $\left[\left(m_{i L}, n_{i L}\right),\left(m_{i H}, n_{i H}\right)\right]$. Of course, it could be that $\left(m_{i L}, n_{i L}\right)=\left(m_{i H}, n_{i H}\right)$, in which case the health plan would de facto be offering a single contract. Hence offering a single contract can be seen as a particular case of offering a menu. Whether this can be sustained as an equilibrium or not is one of our main questions. To ease notation, let $w_{i J}=\left(m_{i J}, n_{i J}\right)$ for all $i=1,2, \ldots, N$ and $J=L, H$. We also use $W_{i}=\left(w_{i L}, w_{i H}\right)$ and $\mathcal{W}=\left(W_{i}\right)_{i=1}^{N}$.

\subsection{Agent's decisions and demands}

Each health plan offers two (possibly different) contracts. Let $U_{i}^{J *}=\operatorname{Max}\left\{U^{J}\left(w_{i L}\right)\right.$, $\left.U^{J}\left(w_{i H}\right)\right\}$ for all $i=1, \ldots, N$ and $J=L, H$. The details of how one derives the demands for each health plan can be found in Chen and Riordan (2007). For our case where each and all spokes have exactly one health plan, the unweighted demand coming from type- $J$ agents is given by

$$
D_{i J}(\mathcal{W})=\frac{1}{N-1} \frac{2}{N} \sum_{k \neq i}\left\{\begin{array}{cl}
0 & \text { if } U_{i}^{J *} \leq U_{k}^{J *}-t \\
\frac{U_{i}^{J *}-U_{k}^{J *}+t}{2 t} & \text { if } U_{k}^{J *}-t \leq U_{i}^{J *} \leq U_{k}^{J *}+t \\
1 & \text { if } U_{i}^{J *}>U_{k}^{J *}+t .
\end{array}\right.
$$

To gain some intuition on this formula, we provide a few (extreme) examples. Fix some type $J$ and look at health plan $i^{\prime}$ s demand as a proportion within this type's population. Suppose first that all plans offer the same final utility to type $J$, i.e., $U_{i}^{J *}=U_{k}^{J *}$

\footnotetext{
13 This is also standard in the literature, again with the exception of Reggiani (2009). In any case, if locations and alternative plans were verifiable and contracts could discriminate accordingly, we would return to the standard (homogeneous product) adverse selection model for each location and alternative plan configuration. This would not add new insights to the homogenous product model.
} 
for all $k$. We have $D_{i J}(\mathcal{W})=\frac{1}{N-1} \frac{2}{N}(N-1) \frac{1}{2}=\frac{1}{N}$, as expected. Suppose now that the best contract for type $J$ out of those offered by $i$ is so much inferior to the rivals' that $U_{i}^{J *} \leq U_{k}^{J *}-t$ for all $k$. Then even individuals residing at the extreme of health plan $i$ 's spoke would prefer to travel to the randomly assigned alternative, no matter where it is, and therefore $D_{i J}(\mathcal{W})=0$. Finally, suppose that $U_{i}^{J *} \geq U_{k}^{J *}+t$ for all $k$. Then all individuals, no matter where they reside, would prefer to travel to the health plan in spoke $i$ if that was indeed a possibility. A proportion $\frac{N-1}{N}$ of the population of the given type resides outside $i$ 's spoke, and out of these, a proportion $1 /(N-1)$ are lucky enough to be given the possibility to enroll plan $i$. This yields a compounded proportion of $\frac{N-1}{N} \frac{1}{N-1}=\frac{1}{N}$ outsiders. A proportion $1 / N$ of this type's population resides in spoke $i$, and they can and will enroll with health plan $i$. This yields a proportion of insiders equal to $1 / N$. The sum of outsiders plus insiders yields $2 / N$, as given by the formula: $\frac{1}{N-1} \frac{2}{N} \sum_{k \neq i} 1=\frac{1}{N-1} \frac{2}{N}(N-1)=\frac{2}{N}$.

Respectively, $\gamma D_{i L}(\mathcal{W})$ and $(1-\gamma) D_{i H}(\mathcal{W})$ are the demands of type $L$ and $H$ that health plan $i$ faces. To ease notation, we omit $(\mathcal{W})$ from the notation in demand. It is clear that each health plan demands depend on all the outstanding contracts in the economy. Since agents receive treatment for free, we do not need to impose any liquidity or participation constraints. ${ }^{14}$

Most importantly, notice that this model reduces to the usual Hotelling model with maximum differentiation when $N=2$.

\subsection{Health plans' payoffs}

The per capita profit obtained by any health plan that has attracted an agent of type $J$ with contract $w=(m, n)$ is given by

$$
\Pi_{J}(w)=r-m-p_{J} n, \quad J=L, H .
$$

If health plan $i$ offers contracts $w_{i L}=\left(m_{i L}, n_{i L}\right)$ and $w_{i H}=\left(m_{i H}, n_{i H}\right)$, then its average payoff is given by:

$$
\gamma D_{i L} \Pi_{L}\left(w_{i L}\right)+(1-\gamma) D_{i H} \Pi_{H}\left(w_{i H}\right)
$$

where $D_{i L}$ and $D_{i H}$ are given in 2. Therefore, we are assuming that health plans are risk neutral.

\subsection{Efficiency and pre-efficiency}

Notice that productive efficiency would require that the marginal benefit of one dollar invested in any given treatment should be equated to its marginal cost, which is of course 1 . Hence a contract $w=(m, n)$ is efficient if $v^{\prime}(m)=v^{\prime}(n)=1$. Notice that a necessary but not sufficient condition for efficiency is that $v^{\prime}(m)=v^{\prime}(n)$, implying

\footnotetext{
14 If transportations costs were very large, patients might decide not to take any treatment. It is enough to assume that $v(0)=-\infty$ to avoid this possibility.
} 
$m=n$, a condition that we refer to as pre-efficiency. If a contract is pre-efficient, any departure from it will either lead to lower profits or make the individual worse-off (all in expected terms). It turns out that whether pre-efficiency is satisfied or not is important for some arguments below. ${ }^{15}$

Definition 1 If a contract $w=(m, n)$ satisfies $m=n$, i.e., if quality across treatments is equalized, we then say that contract $w$ is pre-efficient.

\subsection{The timing of the game}

The first mover is Nature, who chooses both each agent's type according to $\operatorname{Pr}(L)=\gamma$ and each agent's alternative plan $k$ according to $\operatorname{Pr}(k \mid$ reside in spoke $i)=1 /(N-1)$ for all $i$ and $k \neq i$. The second movers are the health plans, who simultaneously offer a contract or a menu of contracts without any capacity constraints. Once a contract is offered, it cannot be withdrawn. Nor can it be denied to an agent demanding it. That is, for all $i=1, \ldots, N$; health plan $i$ commits to serve any agent willing to accept a contract $w$ at health plan $i$. The last movers are the agents, who decide which contract to accept out of those outstanding in the market.

\subsection{Equilibrium}

We define equilibrium next, applying the notion of subgame perfection on the timing described above. ${ }^{16}$ The equilibrium of the subgame played by agents yields the demand functions given in 2. Given these demands, health plans simultaneously choose contracts. We restrict attention to pure-strategy equilibria.

Definition 2 A vector of menus of contracts $\left\{\left[w_{i L}, w_{i H}\right]\right\}_{i=1}^{N} \in\left(\Re^{2} \times \Re^{2}\right)^{N}$ with associated demands defined in 2 is said to be an equilibrium if neither health plan gains additional profits by offering an alternative menu of contracts $\left[w_{L}^{\prime}, w_{H}^{\prime}\right]$, given the menu of contracts offered by the other health plans.

\subsection{The equilibrium in the homogeneous product case}

As a benchmark, we first characterize the (unique) equilibrium set of contracts $\left\{w_{L}^{0}, w_{H}^{0}\right\}$ when there is no differentiation $(t=0)$. In order to derive a parallelism with Rothschild and Stiglitz (1976) model of the market for insurance with free

\footnotetext{
15 If the valuation function was different across treatments then a contract $(m, n)$ would be efficient if $v_{M}^{\prime}(m)=v_{N}^{\prime}(n)=1$ and it would be pre-efficient if $n=\phi(m)$ for some increasing function $\phi$. Since we have assumed that $v_{M}(z)=v_{N}(z)$ for all $z, \phi$ is the identity here.

16 Incidentally, in the homogeneous product case, several authors (Miyazaki 1977 and Spence 1978 among others) have used Wilson (1977) equilibrium notion to derive their results. According to this equilibrium notion, when one calculates the payoff of a deviation, the deviating firm assumes that other firms will withdraw those insurance contracts that generate losses due to the deviation. Riley (2001) and Crocker and Snow (1985) criticize these non-Nash behavioral assumptions. We instead stick to the standard Nash equilibrium concept.
} 
entry, we assume that there are at least three health plans in the market, $N \geq 3 .{ }^{17}$ The equilibrium set of contracts, if it exists, solves the following system of four equations with four unknowns $\left(m_{H}^{0}, n_{H}^{0}, m_{L}^{0}, n_{L}^{0}\right)$ :

(i) Zero profits from the high risks, that is $\Pi_{H}\left(w_{H}^{0}\right)=r-m_{H}^{0}-p_{H} n_{H}^{0}=0$,

(ii) Efficiency for the high risk contract, or $m_{H}^{0}=n_{H}^{0}$,

(iii) Zero profits from the low risks, or $\Pi_{L}\left(w_{L}^{0}\right)=r-m_{L}^{0}-p_{L} n_{L}^{0}=0$, and

(iv) High risks' binding incentive compatibility constraint, or

$$
U^{H}\left(w_{H}^{0}\right)=v\left(m_{H}^{0}\right)+p_{H} v\left(n_{H}^{0}\right)=v\left(m_{L}^{0}\right)+p_{H} v\left(n_{L}^{0}\right)=U^{H}\left(w_{L}^{0}\right)
$$

The equilibrium is obtained graphically in Fig. 3. The per capita isoprofit lines are straight and their slope is $-1 / p_{J}, J=H, L$. The indifference curves have slope $-v^{\prime}(m) / p_{J} v^{\prime}(n), J=H, L$. Pre-efficient contracts lie in the $45^{\circ}$ line, where $m=n$ and the slopes are equated. Equations (i) and (ii) yield $w_{H}^{0}$. Then use (iii) and (iv) to obtain $w_{L}^{0}$. Notice that the horizontal intercept of the zero-isoprofits for both types is $r$, since $r-m_{J}-p_{H} n_{J}=0$ and $n_{J}=0$ imply $m_{J}=r$, for all $J=H, L$. The indifference curve (v) will be used later on.

Existence is guaranteed if the set of contracts $\left\{w_{L}^{0}, w_{H}^{0}\right\}$ is second best, that is, if it is impossible to implement a Pareto-improvement unless the types of individuals are observable to the planner. ${ }^{18}$ This is in turn guaranteed if the proportion $\gamma$ of low risks is not too high. ${ }^{19}$ We assume that this is the case for the rest of the analysis.

It is important to point out that, despite a single equilibrium set of contracts exists, there are many equilibrium configurations. As explained in the introduction, one is where health plans fully specialize. That is, each of the $N$ health plans offers exactly one of the contracts in the set $\left\{w_{L}^{0}, w_{H}^{0}\right\}$. Formally,

Proposition 1 Suppose that $N>2$ and that $\left\{w_{L}^{0}, w_{H}^{0}\right\}$ is second best. Then the following allocation of contracts and agents to health plans constitutes an equilibrium. Health plans belong to exactly one of the following two sets: the set L of health plans that offer the single contract $w_{L}^{0}$ and only attract low risks, or the set $H$ of health plans

17 As pointed out by Villeneuve (2003), if there are only two health plans then the equilibrium with full specialization breaks down. The argument is simple and makes use of Fig. 3. If (WLOG) health plan 1 offers $w_{H}^{0}$ and health plan 2 offers $w_{L}^{0}$, then health plan 2 can deviate to a contract $w_{L}^{\prime}$ slightly to the South West of $w_{L}^{0}$ (not depicted). Despite the fact that such a contract is worse than $w_{L}^{0}$ in all dimensions, it still attracts all low risks. This is for two reasons. First, no health plan offering $w_{L}^{0}$ remains. Second, the low risk incentive compatibility constraint is not binding in the putative equilibrium, so $U^{L}\left(w_{H}^{0}\right)<U^{L}\left(w_{L}^{0}\right)$. Since $U^{L}\left(w_{L}^{\prime}\right)$ can be as close to $U^{L}\left(w_{L}^{0}\right)$ as desired, we have $U^{L}\left(w_{H}^{0}\right)<U^{L}\left(w_{L}^{\prime}\right)$. Notice that $w_{L}^{\prime}$ brings additional profits per low risk. Finally and most importantly, $w_{L}^{\prime}$ does not attract any high risks since it lies below the high risk indifference curve going though $w_{H}^{0}$. Hence the deviation brings additional profits. Part of this argument points at the sufficiency of having three health plans to sustain the equilibrium with full specialization: if $N \geq 3$ then one health plan offers $w_{H}^{0}$ and the other two offer $w_{L}^{0}$. An unilateral deviation to $w_{L}^{\prime}$ attracts no one.

18 Technically, second best optimality is sufficient to guarantee existence of a competitive equilibrium. It would not be necessary if firms were constrained to offer single contracts. Indeed, as shown by Rothschild and Stiglitz (1976), competitive equilibria exist that are not second best if $\gamma$ takes some intermediate interval of values. If one instead allows firms to offer menus (as we do), it is still an open question whether second best optimality is necessary for existence or not.

19 This was proven by Rothschild and Stiglitz (1976). 


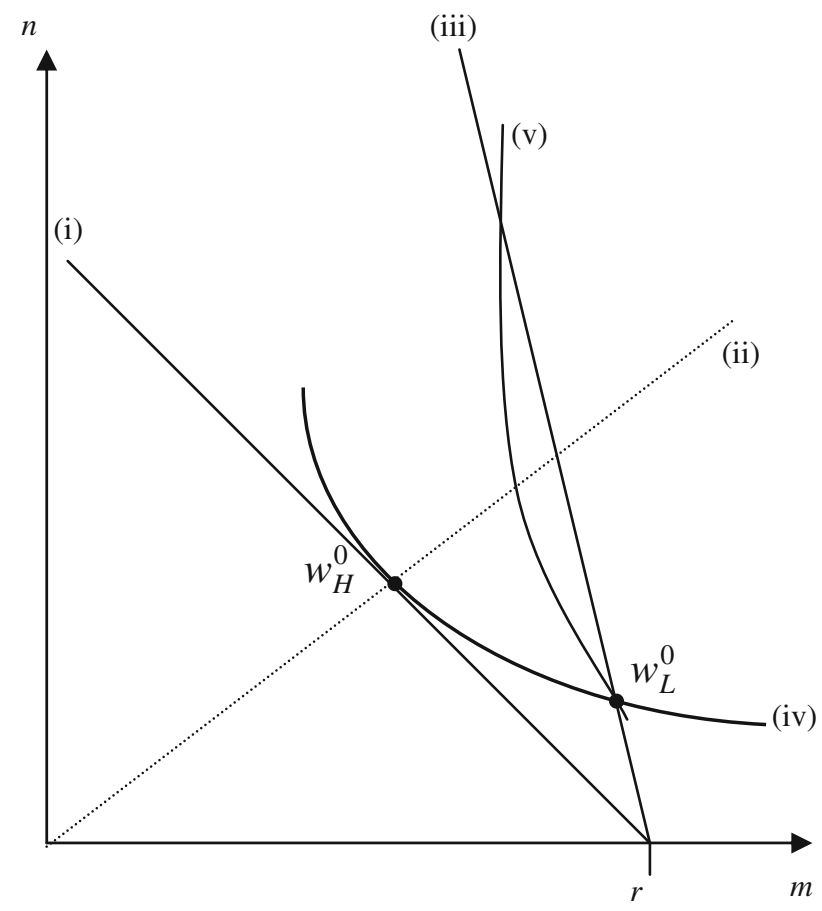

Fig. 3 The set of equilibrium contracts under homogeneous product

that only offer the single contract $w_{H}^{0}$ and only attract high risks; moreover, there are at least 2 health plans in set $L$ and there is at least one health plan in set $H$.

It is important to underline that the equilibrium with full specialization is obtained despite the fact that we are allowing health plans to offer menus of contracts. In the next section we prove our most important result, namely, that under horizontal differentiation, however small, we can rule out this equilibrium. Also in the next section we rule out any configuration where all health plans attract both types of agents with a single contract, so that a minimum contract complexity prevails in equilibrium.

\section{The game under differentiation}

We start with our main contribution, i.e., ruling out equilibria with full specialization.

Proposition 2 Assume that $t>0$, however small. Then there does not exist an equilibrium where health plans are fully specialized, that is, where some health plans only attract high risks with a single contract and the rest of health plans only attract low risks with another single contract while all risks are served. ${ }^{20}$

\footnotetext{
${ }^{20}$ Notice that the configuration that we are ruling out would have to involve two different contracts. Suppose, by contradiction, that all health plans offer the same single contract. Then, since both types are distributed uniformly on the spokes, such contract would attract an equal share of high and low risks, contradicting full specialization.
} 
Let us make use of a particular example to provide some intuition. Take $N=5$. Suppose that $w_{i}$ is offered by health plans 1,2 and 3 and that they so attract all low risks and suppose that $w_{k}$ is offered by health plans 4 and 5 and that they so attract all high risks. This means that high risks close to 1,2 , or 3 and who have health plan 4 or 5 as an alternative prefer $w_{k}$ to $w_{i}$, despite transportation costs. Symmetrically, low risks close to 4 or 5 and who have health plan 1, 2 or 3 as an alternative prefer $w_{i}$ to $w_{k}$ despite transportation costs. Hence, the pair of contracts $\left\{w_{i}, w_{k}\right\}$ satisfies all types' incentive compatibility constraints with some slack. Suppose that either health plan 4 (or 5) unilaterally deviates by offering the menu $\left\{w_{i}, w_{k}\right\}$. Then this health plan still captures his share of high risks and steals all low risks on its spoke from health plans 1,2 , and 3 . Moreover, health plan $k$ is able to do this without violating incentive compatibility. Its profits increase if $\Pi_{L}\left(w_{i}\right)$ is positive, which we can guarantee thanks to the existing market power that differentiation brings. The formal proof extends this intuition to having any number of health plans and to any configuration of contracts.

As explained in the introduction, another important contribution of our analysis is to provide support for the strategy of focusing on the symmetric separating equilibrium once differentiation is present. The next proposition provides this support as it rules out an important class of potential equilibrium configurations, basically, those where single contracts are used rather than menus.

Proposition 3 Suppose that in equilibrium all health plans attract agents of both types (that is, $D_{i J}>0$ for all $i=1, \ldots, N$ and $J=L, H$ ). Then at least one health plan must be offering a menu of contracts. ${ }^{21}$

Thus, we can ensure that interior equilibria must entail some minimum contract complexity, in the sense explained in the introduction. The intuition behind this proposition follows two steps. The first step is to understand that if a health plan attracts a positive quantity of both types in equilibrium with a single contract, this contract must be pre-efficient $(m=n)$. Suppose it is not. This same health plan can increase its profits by deviating to a separating menu of contracts. This separating menu offers the low risk the same non-pre-efficient contract while it offers the high risk the contract that is pre-efficient and gives him the same utility as the original contract. It turns out that such a menu of contracts is incentive compatible. The deviating health plan makes additional per capita profits on the high risk while his demand of both types remains unaltered. In other words, pre-efficiency is a necessary condition for a contract to be robust to menu deviations by the same health plan (internal stability).

The second step is to understand that pre-efficiency in one health plan does not imply that all rival's best response are also a pre-efficient contract. Notice that pre-efficiency puts constraints on the contract. Once a health plan offers a single pre-efficient contract, another health plan's best response is not another pre-efficient contract (external

\footnotetext{
${ }^{21}$ Notice we are also ruling out that all health plans offer the same single contract. If this was the case then, since both types are distributed uniformly on the spokes, such a contract would be attracting both types. This would imply that the assumptions in Proposition 3 apply (all health plans attract both types of agents with a single contract each). But Proposition 3 says that at least one health plan would have to be offering a menu, contradiction. Notice also that Proposition 3 is not a generalization of Proposition 2 because the premise in Proposition 3 is that each and all health plans attract agents of both types, which fails to hold under full specialization.
} 
stability fails). The reason is that the rival health plan will try to screen agents in order to avoid the high risks. Therefore, a vector of $N$ pre-efficient contracts (one per health plan) attracting a positive quantity of both types is not an equilibrium. But if one of the contracts is not pre-efficient then the health plan offering it can do better by deviating to a menu (by step 1). This explains Proposition 3.

\section{Concluding remarks}

We have shown that, even if firms are allowed to offer menus of contracts in a model of the market for health plans, an equilibrium with full specialization cannot be ruled out in the perfectly competitive setting with more than two health plans. Full specialization entails each health plan offering a single contract in each risk class, although the market induces separation because different health plans offer different contracts. More importantly, we have also shown that this equilibrium is non-generic, since any horizontal differentiation would rule it out. In other words, at least one health plan should be attracting more than one type of agent. Another contribution of our analysis is to rule out another large class of equilibria, namely, those where health plans offer a single contract each and yet attract both types of agents. This constitutes a lower bound on the complexity of contracts at each health plan and for each risk class.

One may wonder why there are instances of markets in reality where no health plan offers a menu. Generally speaking, there are several possible answers to this, some coming from our analysis, some lying beyond its scope. First, if plans are homogeneous (that is, if "transportation costs" are nil) then we have shown that it is possible to sustain an equilibrium where no health plan offers a menu and where plans specialize in attracting just one type of agent. However, as mentioned above, this a very non-generic result. Another possibility would be that agents do not have any private information, that is, that the plans' classification system is as fine a partition of as the one that agent's information conveys. ${ }^{22}$ Outside the scope of our analysis is the possibility that maintaining a menu of contracts might be costly, as it would entail checking, at every point of use, which of the contracts in the menu has the enrollee signed for. ${ }^{23}$ Finally, it could also be the case that laws exist that forbid menus altogether, as the MUFACE system serving health care to civil servants in Spain. ${ }^{24}$

Turning back to our setting, our results provide partial support to focusing on the symmetric separating equilibrium where each health plan offers the same menu of incentive compatible contracts, which is the method used in the literature. The discussion above suggests that, even in the light of our results, such a method could be misleading in the presence of regulations that limit the flexibility, assumed in many analyses, that plans have in designing their contracts.

\footnotetext{
22 This is a highly controversial issue. See for instance, Finkelstein and McGarry 2006 and references thereof.

23 Newhouse (1996); Miravete (2009).

24 We are grateful to a referee for pointing this out to us. The MUFACE case is quite extreme, because regulation not only bans menus but also mandates that, in each service, the quality served by the private health plans must be equivalent to that of the public health system (INSS). Inspections are performed regularly to ensure this.
} 
Acknowledgments The authors would like to thank William Encinosa, Izabela Jelovac, Inés MachoStadler, David Pérez-Castrillo, two anonymous referees and the Editor for their comments and suggestions. We greatly benefited from comments by Javier Hernández on the Spanish MUFACE case. Any remaining errors are the authors' responsibility. Vera acknowledges financial support by a Marie Curie Fellowship under contract no. HPMF-CT-01206. Olivella acknowledges financial support from projects SEJ200600538, ECO2009-7616, Consolider-Ingenio CSD2006-16, 2009SGR-169, and Barcelona EconomicsXarxa CREA. Olivella is a Research Fellow of MOVE (Markets, Organizations and Votes in Economics).

Open Access This article is distributed under the terms of the Creative Commons Attribution Noncommercial License which permits any noncommercial use, distribution, and reproduction in any medium, provided the original author(s) and source are credited.

\section{Appendix}

Proof of Proposition 1 It is obvious that no health plan gains by offering the menu $\left\{w_{L}^{0}, w_{H}^{0}\right\}$, since both $\Pi_{L}\left(w_{L}^{0}\right)$ and $\Pi_{H}\left(w_{H}^{0}\right)$ are zero. Now suppose that some health plan deviates to an alternative menu $\left\{w_{L}^{\prime}, w_{H}^{\prime}\right\}$.

Suppose first that the menu only attracts low risks and does so through $w_{L}^{\prime}$. For $w_{L}^{\prime}$ to attract low risks it must be true that $w_{L}^{\prime}$ is on or above curve (v) in Fig. 1, or

$$
U^{L}\left(w_{L}^{\prime}\right) \geq U^{L}\left(w_{L}^{0}\right) .
$$

The reason is that there are at least two health plans offering contract $w_{L}^{0}$. For this deviation to make additional—and hence positive-profits, contract $w_{L}^{\prime}$ must be strictly below isoprofit line $\Pi_{L}(w)=0$ (given by (iii)). Hence $w_{L}^{\prime}$ is in the lens formed by (v) and (iii) and not coincide with $w_{L}^{0}$. Notice that this implies that the high risks strictly prefer $w_{L}^{\prime}$ to $w_{H}^{0}$, or

$$
U^{H}\left(w_{L}^{\prime}\right)>U^{H}\left(w_{H}^{0}\right) .
$$

This contradicts that the menu $\left\{w_{L}^{\prime}, w_{H}^{\prime}\right\}$ only attracts low risks.

Suppose now that the menu $\left\{w_{L}^{\prime}, w_{H}^{\prime}\right\}$ only attracts high risks, and that it does so through $w_{H}^{\prime}$. The high risks have always the option to accept $w_{L}^{0}$, which by binding incentive compatibility of the original set of contracts, is as good as $w_{H}^{0}$, i.e.,

$$
U^{H}\left(w_{L}^{0}\right)=U^{H}\left(w_{H}^{0}\right) .
$$

Hence, even if the deviating health plan was the only one that was originally offering $w_{H}^{0}$, to attract high risks it must be true that $w_{H}^{\prime}$ is above curve (iv) in Fig. 1, or

$$
U^{H}\left(w_{H}^{\prime}\right) \geq U^{H}\left(w_{H}^{0}\right) .
$$

By tangency, at $w_{H}^{0}$, of the isoprofit line $\Pi_{H}(w)=0$ [given by (i)] and the indifference curve $U^{H}(w)=U^{H}\left(w_{H}^{0}\right)$ [given by (iv)], $w_{H}^{\prime}$ must yield losses, so such deviation is not profitable.

Finally, suppose that the menu $\left\{w_{L}^{\prime}, w_{H}^{\prime}\right\}$ is incentive compatible and attracts both types. Then both A1 and A4 must hold. For such a deviation to be profitable it must be true that 


$$
\gamma \Pi^{L}\left(w_{L}^{\prime}\right)+(1-\gamma) \Pi^{H}\left(w_{H}^{\prime}\right)>0 .
$$

Notice that A1, A4, and A5 jointly imply that the set of contracts $\left\{w_{L}^{0}, w_{H}^{0}\right\}$ is not second best: health plans strictly prefer $\left\{w_{L}^{\prime}, w_{H}^{\prime}\right\}$ to $\left\{w_{L}^{0}, w_{H}^{0}\right\}$ while individuals are at least as well-off; a contradiction.

\section{Proof of Proposition 2 In several steps.}

Step 1 Definitions. For each $J \in\{L, H\}$, suppose a non-empty set of health plans $I_{J}$ only attracts $J$-type individuals, and that each health plan $i \in I_{J}$ is doing so by means of contract $\left(m_{i}^{J}, n_{i}^{J}\right)$. Suppose also that $I_{L} \cup I_{H}=I$ and $I_{L} \cap I_{H}=\varnothing$. Let $w_{-i}=\left[\left(m_{1}^{J}, n_{1}^{J}\right), \ldots,\left(m_{i-1}^{J}, n_{i-1}^{J}\right),\left(m_{n+1}^{J}, n_{i+1}^{J}\right), \ldots,\left(m_{N}^{J}, n_{N}^{J}\right)\right]$, where $J$ is to be substituted by the appropriate type depending on whether $i \in I_{L}$ or $i \in I_{H}$.

Step 2 Strict incentive compatibility. The fact that no health plan $i$ in $I_{L}$ attracts any high risk implies that even the high risk that is closest to health plan $i$ prefers to travel the full length of $i$ 's spoke plus the full length of the spoke of alternative health plan $k \in I_{H}$ than enrolling health plan $i$. Formally, $v\left(m_{k}^{H}\right)+p_{H} v\left(n_{k}^{H}\right)-t \geq v\left(m_{i}^{L}\right)+p_{H} v\left(n_{i}^{L}\right)$ for all $k \in I_{H}$ and $i \in I_{L}$. Similarly, the fact that no health plan $k$ in $I_{H}$ attracts any low risk implies that $v\left(m_{i}^{L}\right)+p_{L} v\left(n_{i}^{L}\right)-t \geq v\left(m_{k}^{H}\right)+p_{H} v\left(n_{k}^{H}\right)$ for all $k \in I_{H}$ and $i \in I_{L}$.

Step 3 Show that all health plans in $I_{L}$ make positive profits. Suppose first that one health plan makes negative profits. Then let this health plan deviate to $m=n=0$. Hence $r-m-p_{L} n=r>0$. The worst that can happen is that this health plan attracts no-one. In that case the health plan still makes zero profits, a contradiction. Let us suppose mow, by contradiction, that some health plan $i$ in $I_{L}$ makes exactly zero profits, or $r-m_{i}^{L}-p_{J} n_{i}^{J}=0$. Let this health plan deviate from $\left(m_{i}^{L}, n_{i}^{L}\right)$ to $w^{\prime}=$ $\left(m^{\prime}, n^{\prime}\right)=\left(m_{i}^{J}-\varepsilon, n_{i}^{J}-\varepsilon\right)$. If health plan $i$ was not attracting any high risks with the original contract, less it will do so with a worse contract. Hence $D_{i H}\left(w^{\prime}, w_{-i}\right)=0$. As for the low risks' demand, this will be given by

$$
\begin{aligned}
& D_{i L}\left(w^{\prime}, w_{-i}\right)= \\
& \frac{1}{N-1} \frac{2}{N} \sum_{\substack{k \neq i \\
k \in I_{L}}} \frac{t+v\left(m_{i}^{L}-\varepsilon\right)+p_{L} v\left(n_{i}^{L}-\varepsilon\right)-v\left(m_{k}^{L}\right)-p_{L} v\left(n_{k}^{L}\right)}{2 t} \\
& \stackrel{\text { def }}{=} \widetilde{D}_{L}(\varepsilon) .
\end{aligned}
$$

Now, since $i$ was attracting some $L$ individuals in the original contract profile, it must have been true that $\widetilde{D}_{L}(0)>0$. Hence, by continuity of the function $\widetilde{D}_{L}$, there must exist $\varepsilon>0$ such that $\widetilde{D}_{L}(\varepsilon)>0$. Hence health plan $i$ 's total profits are increased by $\gamma \widetilde{D}_{L}(\varepsilon)\left(r-m_{i}^{L}+\varepsilon-p_{L} n_{i}^{L}+p_{L} \varepsilon\right)=\gamma \widetilde{D}_{L}(\varepsilon) \varepsilon\left(1+p_{L}\right)>0$, and we have found a profitable deviation.

Step 4 Construct a profitable deviation from the putative equilibrium. Pick any health plan in $I_{L}$, say $i^{*}$ and any health plan $k^{*}$ in $I_{H}$. Now assume that health plan $k^{*}$, instead 
of offering contract $\left(m_{k^{*}}^{H}, n_{k^{*}}^{H}\right)$, offers the menu $\left\{\left(m_{k^{*}}^{H}, n_{k^{*}}^{H}\right),\left(m_{i^{*}}^{L}, n_{i^{*}}^{L}\right)\right\}$. By Step 2, $v\left(m_{k^{*}}^{H}\right)+p_{H} v\left(n_{k^{*}}^{H}\right)-t \geq v\left(m_{i}^{L}\right)+p_{H} v\left(n_{i}^{L}\right)$ for all $i \in I_{L}$ (including $\left.i^{*}\right)$, so the first contract in the menu will only attract high risks, and health plan $k^{*}$ 's share of high risks will remain the same, as well as the profits per high risk individual. Also by Step 2, $v\left(m_{i^{*}}^{L}\right)+p_{L} v\left(n_{i^{*}}^{L}\right)-t \geq v\left(m_{k}^{H}\right)+p_{H} v\left(n_{k}^{H}\right)$ for all $k \in I_{H}$, so the second contract in the menu will only attract low risks. The question is whether it will attract any low risks. If it does we are done since these affiliates are new for health plan $k$, and in Step 3 we showed that such consumers bring in positive profits per capita. Consider low risks who reside in health plan $k^{*}$ 's spoke. Nature assigns to some of them health plan $i^{*}$ as the only alternative health plan. Such individuals have to choose between health plan $k^{*}$ 's contract aimed to low risks and the same contract offered by health plan $i^{*}$. They will of course choose to stay with the closer health plan $k^{*}$, and we are done.

\section{Proof of Proposition 3}

Step 1 If a health plan $i$ offers a single contract $\left(m_{i}, n_{i}\right)$ (or two contracts of which one is never chosen) and if $0<D_{i L}=D_{i H}<1$, then this health plan's contract must be pre-efficient. That is, $m_{i}=n_{i}$.

Assume first, by contradiction, that a health plan offers the contract $w^{\prime}=\left(m^{\prime}, n^{\prime}\right)$ with $m^{\prime}>n^{\prime}$. We now find an alternative strategy that increases the profits of this health plan, whatever the other health plan does. Consider the menu of contracts $\left[w^{\prime}, w^{\prime \prime}\right]$, with $m^{\prime}>m^{\prime \prime}=n^{\prime \prime}>n^{\prime}$, and $U^{H}\left(w^{\prime \prime}\right)=U^{H}\left(w^{\prime}\right)$. Hence $H$ has no interest in camouflaging when asked to choose $w^{\prime \prime}$. We prove next that $L$ has no interest in camouflaging when asked to choose $w^{\prime}$. Notice first that $U^{H}\left(w^{\prime \prime}\right)=U^{H}\left(w^{\prime}\right)$ implies that $v\left(n^{\prime}\right)=\frac{v\left(m^{\prime \prime}\right)-v\left(m^{\prime}\right)}{p_{H}}+v\left(n^{\prime \prime}\right)$. Hence $U^{L}\left(w^{\prime}\right)-U^{L}\left(w^{\prime \prime}\right)=v\left(m^{\prime}\right)+p_{L} v\left(n^{\prime}\right)-$ $v\left(m^{\prime \prime}\right)-p_{L} v\left(n^{\prime \prime}\right)=v\left(m^{\prime}\right)+p_{L}\left[\frac{v\left(m^{\prime \prime}\right)-v\left(m^{\prime}\right)}{p_{H}}+v\left(n^{\prime \prime}\right)\right]-v\left(m^{\prime \prime}\right)-p_{L} v\left(n^{\prime \prime}\right)=(1-$ $\left.\frac{p_{L}}{p_{H}}\right)\left[v\left(m^{\prime}\right)-v\left(m^{\prime \prime}\right)\right]>0$. Notice that both types are indifferent between the previous single contract and the new menu of contracts. Therefore, demands are unaltered whatever the type. Since $D_{i H}>0$, it suffices to show that profits derived from one $H$-agent are larger. (The profits derived from the other type are unaltered.) This is straightforward: contracts $w^{\prime}$ and $w^{\prime \prime}$ yield the same expected utility to agents while $w^{\prime \prime}$ is pre-efficient while $w^{\prime}$ is not.

A similar argument can be used to show that a pooling contract $w^{\prime}=\left(m^{\prime}, n^{\prime}\right)$ with $m^{\prime}<n^{\prime}$, cannot be part of a pooling equilibrium. (Use a menu of contracts where $L$ receives a pre-efficient contract without changing his utility and which leaves the original pooling unaltered. The high risk will stay put and the low risk will move to the new contract. Profits derived from the low risk increase.)

Step 2 If in equilibrium $0<D_{i L}<1$ and $0<D_{i H}<1$ for all $i$, then it cannot be the case that all health plans offer a single contract each.

Suppose that $0<D_{i L}<1$ and $0<D_{i H}<1$ but that, by contradiction, all health plans offer a single contract each, say $\bar{w}_{i}=\left(\bar{m}_{i}, \bar{n}_{i}\right)$. By step 1 , this implies that $\bar{m}_{i}=\bar{n}_{i}$ for all $i$. Since this constitutes an equilibrium then it must be the case that $\bar{W}_{i}=\left(\bar{w}_{i}^{H}, \bar{w}_{i}^{L}\right)=\left[\left(\bar{m}_{i}, \bar{m}_{i}\right),\left(\bar{m}_{i}, \bar{m}_{i}\right)\right]$ is health plan $i$ 's best response to each 
of the rest of the health plans' (degenerate menu) choice, ${ }^{25}$ denoted by $\bar{W}_{-i}=$ $\left\{\bar{W}_{k}\right\}_{k \neq i}=\left\{\left(\bar{w}_{k}^{H}, \bar{w}_{k}^{L}\right)\right\}_{k \neq i}=\left\{\left[\left(\bar{m}_{k}, \bar{m}_{k}\right),\left(\bar{m}_{k}, \bar{m}_{k}\right)\right]\right\}_{k \neq i}$. In general, the menu of contracts $W_{i}=\left[\left(m_{i}^{L}, n_{i}^{L}\right),\left(m_{i}^{H}, n_{i}^{H}\right)\right]$ that is the best response to $\bar{W}_{-i}$ solves

$$
\begin{array}{r}
\operatorname{Max} \underset{W_{i}}{\gamma \Pi_{L}\left(m_{i}^{L}, n_{i}^{L}\right) D_{i L}\left(W_{i}, \bar{W}_{-i}\right)} \\
+(1-\gamma) \Pi_{H}\left(m_{i}^{H}, n_{i}^{H}\right) D_{i H}\left(W_{i}, \bar{W}_{-i}\right),
\end{array}
$$

subject to

$$
\begin{aligned}
v\left(m_{i}^{L}\right)+p_{L} v\left(n_{i}^{L}\right) & \geq v\left(m_{i}^{H}\right)+p_{L} v\left(n_{i}^{H}\right) \\
v\left(m_{i}^{H}\right)+p_{H} v\left(n_{i}^{H}\right) & \geq v\left(m_{i}^{L}\right)+p_{H} v\left(n_{i}^{L}\right),
\end{aligned}
$$

where

$$
\begin{aligned}
\Pi_{L}\left(m_{i}^{L}, n_{i}^{L}\right) & =r-m_{i}^{L}-p_{L} n_{i}^{L}, \\
\Pi_{H}\left(m_{i}^{H}, n_{i}^{H}\right) & =r-m_{i}^{H}-p_{H} n_{i}^{H},
\end{aligned}
$$

and, by virtue of the assumption that demands are interior,

$$
\begin{aligned}
& D_{i L}\left(W_{i}, \bar{W}_{-i}\right)=\frac{1}{N-1} \frac{2}{N} \sum_{k \neq i} \frac{v\left(m_{i}^{L}\right)-v\left(\bar{m}_{k}\right)+p_{L}\left(v\left(n_{i}^{L}\right)-v\left(\bar{m}_{k}\right)\right)+t}{2 t} \\
& D_{i H}\left(W_{i}, \bar{W}_{-i}\right)=\frac{1}{N-1} \frac{2}{N} \sum_{k \neq i} \frac{v\left(m_{i}^{H}\right)-v\left(\bar{m}_{k}\right)+p_{H}\left(v\left(n_{i}^{H}\right)-v\left(\bar{m}_{k}\right)\right)+t}{2 t}
\end{aligned}
$$

The Lagrangian is

$$
\begin{aligned}
\mathfrak{£}_{i}= & \gamma \Pi_{L}\left(w_{i L}\right) \frac{1}{N-1} \frac{2}{N} \sum_{k \neq i} \frac{v\left(m_{i}^{L}\right)-v\left(\bar{m}_{k}\right)+p_{L}\left(v\left(n_{i}^{L}\right)-v\left(\bar{m}_{k}\right)\right)+t}{2 t} \\
& +(1-\gamma) \Pi_{H}\left(w_{i H}\right) \frac{1}{N-1} \frac{2}{N} \sum_{k \neq i} \frac{v\left(m_{i}^{H}\right)-v\left(\bar{m}_{k}\right)+p_{H}\left(v\left(n_{i}^{H}\right)-v\left(\bar{m}_{k}\right)\right)+t}{2 t} \\
& \mu_{H}\left(U^{H}\left(w_{i H}\right)-U^{H}\left(w_{i L}\right)\right)+\mu_{L}\left(U^{L}\left(w_{i L}\right)-U^{L}\left(w_{i H}\right)\right) .
\end{aligned}
$$

The Khun and Tucker conditions include (after noting that $\frac{1}{N-1} \frac{2}{N} \sum_{k \neq i} \frac{v^{\prime}\left(m_{i}^{J}\right)}{2 t}=$ $\frac{v^{\prime}\left(m_{i}^{J}\right)}{N t}$ and that $\left.\frac{1}{N-1} \frac{2}{N} \sum_{k \neq i} \frac{v^{\prime}\left(n_{i}^{J}\right)}{2 t}=\frac{v^{\prime}\left(n_{i}^{J}\right)}{N t}\right)$

\footnotetext{
25 The rival's menu is degenerate for two reasons: they are just offering a single contract (or the two components of the menu are the same) and moreover expenditures in the two illnesses is the same.
} 


$$
\begin{aligned}
& \gamma\left[\Pi_{L}\left(w_{i L}\right) \frac{v^{\prime}\left(m_{i}^{J}\right)}{N t}-D_{i L}\right]-\left(\mu_{H}-\mu_{L}\right) v^{\prime}\left(m_{i L}\right)=0, \\
& \gamma p_{L}\left[\Pi_{L}\left(w_{i L}\right) \frac{v^{\prime}\left(m_{i}^{J}\right)}{N t}-D_{i L}\right]-\left(\mu_{H} p_{H}-\mu_{L} p_{L}\right) v^{\prime}\left(n_{i L}\right)=0, \\
& (1-\gamma)\left[\Pi_{H}\left(w_{i H}\right) \frac{v^{\prime}\left(n_{i}^{J}\right)}{N t}-D_{i H}\right]+\left(\mu_{H}-\mu_{L}\right) v^{\prime}\left(m_{i H}\right)=0, \\
& (1-\gamma) p_{H}\left[\Pi_{H}\left(w_{i H}\right) \frac{v^{\prime}\left(n_{i}^{J}\right)}{N t}-D_{i H}\right]+\left(\mu_{H} p_{H}-\mu_{L} p_{L}\right) v^{\prime}\left(n_{i H}\right)=0 .
\end{aligned}
$$

The above conditions must be satisfied at $\left(w_{i}^{H}, w_{i}^{L}\right)=\left[\left(\bar{m}_{i}, \bar{m}_{i}\right),\left(\bar{m}_{i}, \bar{m}_{i}\right)\right]$. Substituting these values into the previous conditions and letting $L=\gamma\left[\Pi_{L}\left(\bar{m}_{i}, \bar{m}_{i}\right)\right.$ $\left.\frac{v^{\prime}\left(\bar{m}_{i}\right)}{N t}-D_{i L}\right]$ and $H=(1-\gamma)\left[\Pi_{H}\left(\bar{m}_{i}, \bar{m}_{i}\right) \frac{v^{\prime}\left(\bar{m}_{i}\right)}{N t}-D_{i H}\right]$ we can write

$$
\begin{aligned}
L & =\left(\mu_{H}-\mu_{L}\right) v^{\prime}\left(\bar{m}_{i}\right), \\
p_{L} L & =\left(\mu_{H} p_{H}-\mu_{L} p_{L}\right) v^{\prime}\left(\bar{m}_{i}\right), \\
H & =-\left(\mu_{H}-\mu_{L}\right) v^{\prime}\left(\bar{m}_{i}\right), \\
p_{H} H & =-\left(\mu_{H} p_{H}-\mu_{L} p_{L}\right) v^{\prime}\left(\bar{m}_{i}\right) .
\end{aligned}
$$

or, adding A6 with A8 and A7 with A9,

$$
\begin{aligned}
L+H & =0, \\
p_{L} L+p_{H} H & =0 .
\end{aligned}
$$

Since $p_{L} \neq p_{H}$, this implies that $L=H=0$, or

$$
\begin{aligned}
\Pi_{L}\left(\bar{m}_{i}, \bar{m}_{i}\right) \frac{v^{\prime}\left(\bar{m}_{i}\right)}{N t} & =D_{i L}, \\
\Pi_{H}\left(\bar{m}_{i}, \bar{m}_{i}\right) \frac{v^{\prime}\left(\bar{m}_{i}\right)}{N t} & =D_{i H} .
\end{aligned}
$$

Now, the above conditions must also hold at any health plan that is best responding to the rest. Hence we can add across $i=1, \ldots, n$ to get

$$
\begin{aligned}
\sum_{i=1, \ldots, n} \Pi_{L}\left(\bar{m}_{i}, \bar{m}_{i}\right) \frac{v^{\prime}\left(\bar{m}_{i}\right)}{N t} & =\sum_{i=1, \ldots, n} D_{i L}=1, \\
\sum_{i=1, \ldots, n} \Pi_{H}\left(\bar{m}_{i}, \bar{m}_{i}\right) \frac{v^{\prime}\left(\bar{m}_{i}\right)}{N t} & =\sum_{i=1, \ldots, n} D_{i H}=1 .
\end{aligned}
$$

Subtract these expressions to get

$$
\sum_{i=1, \ldots, n}\left[\Pi_{L}\left(\bar{m}_{i}, \bar{m}_{i}\right)-\Pi_{H}\left(\bar{m}_{i}, \bar{m}_{i}\right)\right] \frac{v^{\prime}\left(\bar{m}_{i}\right)}{N t}=0 .
$$


However, $\Pi_{L}\left(\bar{m}_{i}, \bar{m}_{i}\right)=r-\bar{m}_{i}\left(1+p_{L}\right)>r-\bar{m}_{i}\left(1+p_{H}\right)=\Pi_{H}\left(\bar{m}_{i}, \bar{m}_{i}\right)$ for all $i$, contradiction. This concludes the proof.

\section{References}

Biglaiser G, Ma CHA (2003) Price and quality competition under adverse selection: market organization and efficiency. RAND J Econ 34:266-286

Chen Y, Riordan MH (2007) Price and variety in the Spokes Model. Econ J 117:897-921

Crocker K, Snow A (1985) A simple tax structure for competitive equilibrium and redistribution in insurance markets with asymmetric information. Southern Econ J 51:1142-1150

Crocker K, Snow A (2000) The theory of risk classification. In: Dionne G (ed) Handbook of insurance. Kluwer, Boston, pp 245-276

Ellis R (1998) Creaming, skimping and dumping: provider competition on the intensive and extensive margins. J Health Econ 17:537-555

Ellis RP, McGuire TG (2007) Predictability and predictiveness in health care spending. J Health Econ 26:25-48

Encinosa W, Sappington D (1997) Competition among health maintenance organizations. J Econ Manage Strategy 6:129-150

Finkelstein A, McGarry K (2006) Multiple dimensions of private information: evidence from the long-term care insurance market. Am Econ Rev 96:938-958

Frank R, Lamiraud K (2009) Choice, price competition and complexity in markets for health insurance. J Econ Behav Organ 71:550-562

Frank R, Glazer J, McGuire T (2000) Measuring adverse selection in managed health care. J Health Econ 19:829-854

Glazer J, McGuire T (2000) Optimal risk adjustment in markets with adverse selection: an application to managed care. Am Econ Rev 90:1055-1071

Jack W (2006) Optimal risk adjustment with adverse selection and spatial competition. J Health Econ 25:908-926

Ma A (2004) Managed care and shadow price. Health Econ 13:199-202

Miravete EJ (2009) Competing with menus of tariff options. J Eur Econ Asso 7:188-205

Miyazaki H (1977) The rate race and internal labor markets. Bell J Econ 8:394-418

Newhouse J (1996) Reimbursing health plans and health providers: efficiency in production versus selection. J Econ Lit 34:1236-1263

Olivella P, Vera-Hernández M (2007) Competition among differentiated health plans under adverse selection. J Health Econ 26:233-250

Olivella P, Schroyen F (2009) Not so shadow prices and optimal risk adjustment. In: Working paper, Universitat Autònoma de Barcelona

Pellisé L (1994) Reimbursing insurance carriers: the case of muface in the Spanish Health Care System. J Health Econ 3:243-253

Reggiani C (2009) Optimal differentiation and spatial competition: the Spokes Model with product differentiation. In: Working paper, University of York

Riley J (2001) Silver signals: twenty-five years of screening and signaling. J Econ Lit 39:432-478

Rothschild M, Stiglitz J (1976) Equilibrium in competitive insurance markets: an essay on the economics of imperfect information. Quart Rev Econ 90:629-649

Salop S (1979) Monopolistic competition with outside goods. Bell J Econ 10:141-156

Spence M (1978) Product differentiation and performance in insurance markets. J Public Econ 10:427-447

Villas-Boas JM, Schmidt-Mohr U (1999) Oligopoly with asymmetric information: differentiation in credit markets. RAND J Econ 30:375-396

Villeneuve B (2003) Concurrence et antisélection multidimensionelle en assurance. Ann Econ Stat 69:119_ 142

Wilson C (1977) A model of insurance markets with incomplete information. J Econ Theory 16:167-207 